\title{
The phase transition lines in pair approximation for the basic reinfection model SIRI
}

\author{
Nico Stollenwerk ${ }^{\mathrm{a}, \mathrm{b}, \mathrm{c}, *}$, José Martins $^{\mathrm{d}}$, Alberto Pinto $^{\mathrm{e}}$ \\ a Instituto Gulbenkian de Ciência, Apartado 14, 2781-901 Oeiras, Portugal \\ ${ }^{\mathrm{b}}$ Research Center Jülich, D-52425 Jülich, Germany \\ ${ }^{\mathrm{c}}$ Centro de Matemática e Aplicações Fundamentais, Faculdade de Ciências, Universidade de Lisboa, Av. Prof. Gama Pinto 2, 1649-003 Lisboa, Portugal \\ d Departamento de Matemática, Escola Superior de Tecnologia e Gestão, Instituto Politécnico de Leiria, Apartado 4163, 2411-901 Leiria, Portugal \\ e Departamento de Matemática Pura, Faculdade de Ciências, Universidade do Porto, Rua do Campo Alegre 687, 4169-007 Porto, Portugal
}

Received 13 March 2007; received in revised form 8 June 2007; accepted 12 June 2007

Available online 21 June 2007

Communicated by C.R. Doering

\begin{abstract}
For a spatial stochastic epidemic model we investigate in the pair approximation scheme the differential equations for the moments. The basic reinfection model of susceptible-infected-recovered-reinfected or SIRI type is analysed, its phase transition lines calculated analytically in this pair approximation.
\end{abstract}

(C) 2007 Elsevier B.V. All rights reserved.

PACS: 87.19.Xx; 05.10.Gg; 05.70.Fh

Keywords: Stochastic processes; Epidemiology; Reinfection model; Pair approximation; Analytics of phase transition lines

\section{Introduction}

Models for reinfection processes in epidemiology have recently attracted interest, especially for a first description of multi-strain epidemics [1,2], where after an initial infection immunity against one strain only gives partial immunity against a genetically close mutant strain. In the physics literature models for partial immunization have also found wide interest $[3,4]$, under somehow different aspects though. The processes investigated by the two scientific communities are closely related [5]. Transitions between no-growth, compact growth and annular growth have been observed in both cases [3,5].

Here we analyse the basic model for reinfection or partial immunization SIRI (susceptible, infected, recovered and again infected) beyond the mean field approximation by considering also pair dynamics and obtain a better qualitative understanding of the process. Namely, in mean field the limiting cases of SIS (susceptible, infected, susceptible), the case of equal primary and secondary infectivity, and SIR (susceptible, infected, recovered), the case of vanishing reinfection, have the same critical value for the transition from no-growth to a nontrivial stationary state. In the case of the SIS model that is the transition from no-growth to compact growth of an area of infection when initially starting with one infected in a susceptible environment. In the case of the SIR model it is the transition from no-growth to annular growth, a ring of infecteds, leaving recovered behind. In pair approximation these critical points have different values, as previously calculated for the SIS system [6] and the SIR system [7]. In addition we calculated the phase transition lines between no-growth and compact growth, between compact growth and annular growth and

\footnotetext{
* Corresponding author at: Research Center Jülich, D-52425 Jülich, Germany.

E-mail address: nks22@cam.ac.uk (N. Stollenwerk).
} 
between no-growth and annular growth analytically, and compare the especially tricky phase transition line between no-growth and annular growth with simulations.

The transition between annular and compact growth has been found for mean field like models under the name of reinfection threshold [1], and has been under debate [2,8]. The geometric interpretation of this threshold in the context of partial immunization [3] clears this debate [5] and the analytic results given here further underline the close relation between the models considered in physics and in epidemiology.

In Section 2 we describe the basic model for reinfection, the SIRI model, in its stochastic description. Deriving dynamic equations for the expectation values of total number of susceptibles, infected and recovered we obtain expressions of pairs, then writing dynamic equations for these we obtain triplets, etc. In Section 3 we find a closed system of dynamic equations for means and pairs via the pair approximation. These equations are analyzed in Section 4 further to obtain the stationary states and critical lines.

\section{The SIRI epidemic model}

We consider the following transitions between host classes for $N$ individuals being either susceptible $S$, infected $I$ by a disease or recovered $R$,

$$
\begin{aligned}
& S+I \stackrel{\beta}{\longrightarrow} I+I, \\
& I \stackrel{\gamma}{\longrightarrow} R, \\
& R+I \stackrel{\tilde{\beta}}{\longrightarrow} I+I, \\
& R \stackrel{\alpha}{\longrightarrow} S,
\end{aligned}
$$

resulting in the master equation [9] for variables $S_{i}, I_{i}$ and $R_{i} \in\{0,1\}, i=1,2, \ldots, N$, for $N$ individuals eventually on a regular grid, with constraint $S_{i}+I_{i}+R_{i}=1$.

The first infection $S+I \stackrel{\beta}{\longrightarrow} I+I$ occurs with infection rate $\beta$, whereas after recovery with rate $\gamma$ the respective host becomes resistant up to a possible reinfection $R+I \stackrel{\tilde{\beta}}{\longrightarrow} I+I$ with reinfection rate $\tilde{\beta}$. Hence the recovered are only partially immunized. For further analysis of possible stationary states we include a transition from recovered to susceptibles $\alpha$, which might be simply due to demographic effects (or very slow waning immunity for some diseases). We will later consider the limit of vanishing or very small $\alpha$. In case of demography that would be in the order of inverse 70 years, whereas for the basic epidemic processes like first infection $\beta$ we would expect inverse a few weeks.

The master equation is of the following form

$$
\begin{aligned}
\frac{d}{d t} p & \left(S_{1}, I_{1}, R_{1}, S_{2}, I_{2}, R_{2}, \ldots, R_{N}, t\right) \\
= & \sum_{i=1}^{N} \beta\left(\sum_{j=1}^{N} J_{i j} I_{j}\right)\left(1-S_{i}\right) p\left(S_{1}, I_{1}, R_{1}, \ldots, 1-S_{i}, 1-I_{i}, R_{i}, \ldots, R_{N}, t\right) \\
& +\sum_{i=1}^{N} \gamma\left(1-I_{i}\right) p\left(S_{1}, I_{1}, R_{1}, \ldots, S_{i}, 1-I_{i}, 1-R_{i}, \ldots, R_{N}, t\right) \\
& +\sum_{i=1}^{N} \tilde{\beta}\left(\sum_{j=1}^{N} J_{i j} I_{j}\right)\left(1-R_{i}\right) p\left(S_{1}, I_{1}, R_{1}, \ldots, S_{i}, 1-I_{i}, 1-R_{i}, \ldots, R_{N}, t\right) \\
& +\sum_{i=1}^{N} \alpha\left(1-R_{i}\right) p\left(S_{1}, I_{1}, R_{1}, \ldots, 1-S_{i}, I_{i}, 1-R_{i}, \ldots, R_{N}, t\right) \\
& -\sum_{i=1}^{N}\left[\beta\left(\sum_{j=1}^{N} J_{i j} I_{j}\right) S_{i}+\gamma I_{i}+\tilde{\beta}\left(\sum_{j=1}^{N} J_{i j} I_{j}\right) R_{i}+\alpha R_{i}\right] p\left(\ldots, S_{i}, I_{i}, R_{i}, \ldots\right) .
\end{aligned}
$$

$J_{i, j} \in\{0,1\}$ are the elements of the $N \times N$ adjacency matrix $J$, symmetric and with zero diagonal elements. The formulation of the master equation is given in analogy to the one used, e.g., in Glauber [10].

The expectation value, e.g., for the total number of infected hosts at a given time is

$$
\langle I\rangle(t):=\sum_{S_{1}=0}^{1} \sum_{I_{1}=0}^{1} \sum_{R_{1}=0}^{1} \sum_{S_{2}=0}^{1} \ldots \sum_{R_{N}=0}^{1}\left(\sum_{i=1}^{N} I_{i}\right) p\left(S_{1}, I_{1}, R_{1}, S_{2}, \ldots, R_{N}, t\right) .
$$


To calculate the dynamics of the moments, mean total number of infected etc., we take the definition of the moments, Eq. (2), and take its time derivative

$$
\frac{d}{d t}\langle I\rangle(t)=\sum_{S_{1}=0}^{1} \sum_{I_{1}=0}^{1} \sum_{R_{1}=0}^{1} \sum_{S_{2}=0}^{1} \cdots \sum_{R_{N}=0}^{1}\left(\sum_{i=1}^{N} I_{i}\right) \frac{d}{d t} p\left(I_{1}, R_{1}, I_{2}, \ldots, R_{N}, t\right) .
$$

Now we have to insert the master equation (1) into Eq. (3) for the expression $\frac{d}{d t} p\left(S_{1}, I_{1}, R_{1}, S_{2}, \ldots, R_{N}, t\right)$ and after some calculation we find the ordinary differential equations (ODEs) in terms of all variables $S, I$ and $R$.

For the mean total number of susceptible, infected and recovered hosts we obtain the following equations

$$
\begin{aligned}
& \frac{d}{d t}\langle S\rangle=\alpha\langle R\rangle-\beta\langle S I\rangle_{1}, \\
& \frac{d}{d t}\langle I\rangle=\beta\langle S I\rangle_{1}-\gamma\langle I\rangle+\tilde{\beta}\langle R I\rangle_{1}, \\
& \frac{d}{d t}\langle R\rangle=\gamma\langle I\rangle-\alpha\langle R\rangle-\tilde{\beta}\langle R I\rangle_{1}
\end{aligned}
$$

which include the pairs, like

$$
\langle S I\rangle_{1}(t):=\sum_{S_{1}=0}^{1} \sum_{I_{1}=0}^{1} \sum_{R_{1}=0}^{1} \cdots \sum_{R_{N}=0}^{1}\left(\sum_{i=1}^{N} \sum_{j=1}^{N}\left(J^{1}\right)_{i j} S_{i} I_{j}\right) p\left(S_{1}, I_{1}, R_{1}, \ldots, R_{N}, t\right) .
$$

In the equation for the dynamics of $\langle S I\rangle_{1}$ also an expression $\langle S I\rangle_{2}$ could show up. These are longer range correlations, formally given by a power of two of the adjacency matrix $J^{2}$ and then its elements $\left(J^{2}\right)_{i j}:=\sum_{k=1}^{N} J_{i k} \cdot J_{k j}$.

We have to calculate equations for the dynamics of the pairs as well. In the equations for the dynamics of pairs the triples, e.g.,

$$
\langle I S I\rangle_{1,1}(t):=\sum_{S_{1}=0}^{1} \sum_{I_{1}=0}^{1} \sum_{R_{1}=0}^{1} \cdots \sum_{R_{N}=0}^{1}\left(\sum_{i=1}^{N} \sum_{j=1}^{N} \sum_{k=1}^{N} J_{i j} J_{j k} I_{i} S_{j} I_{k}\right) p\left(S_{1}, I_{1}, R_{1}, \ldots, R_{N}, t\right)
$$

or equivalently

$$
\langle I S I\rangle_{1,1}(t)=\sum_{i=1}^{N} \sum_{j=1}^{N} \sum_{k=1}^{N} J_{i j} J_{j k}\left\langle I_{i} S_{j} I_{k}\right\rangle
$$

appear, where $\left\langle I_{i} S_{j} I_{k}\right\rangle$ is the local expectation value. After some calculation in analogy to the calculation for the first moments we obtain

$$
\begin{aligned}
& \frac{d}{d t}\langle S S\rangle_{1}=2 \alpha\langle R S\rangle_{1}-2 \beta\langle S S I\rangle_{1,1}, \\
& \frac{d}{d t}\langle I I\rangle_{1}=2 \beta\langle I S I\rangle_{1,1}-2 \gamma\langle I I\rangle_{1}+2 \tilde{\beta}\langle I R I\rangle_{1,1}, \\
& \frac{d}{d t}\langle R R\rangle_{1}=2 \gamma\langle I R\rangle_{1}-2 \tilde{\beta}\langle R R I\rangle_{1,1}-2 \alpha\langle R R\rangle_{1}, \\
& \frac{d}{d t}\langle S I\rangle_{1}=\beta\langle S S I\rangle_{1,1}+\tilde{\beta}\langle S R I\rangle_{1,1}-\gamma\langle S I\rangle_{1}-\beta\langle I S I\rangle_{1,1}+\alpha\langle R I\rangle_{1}, \\
& \frac{d}{d t}\langle R S\rangle_{1}=\gamma\langle S I\rangle_{1}-\beta\langle R S I\rangle_{1,1}-\tilde{\beta}\langle S R I\rangle_{1,1}+\alpha\langle R R\rangle_{1}-\alpha\langle R S\rangle_{1}, \\
& \frac{d}{d t}\langle R I\rangle_{1}=\gamma\langle I I\rangle_{1}+\beta\langle R S I\rangle_{1,1}+\tilde{\beta}\langle R R I\rangle_{1,1}-\gamma\langle I R\rangle_{1}-\tilde{\beta}\langle I R I\rangle_{1,1}-\alpha\langle R I\rangle_{1} .
\end{aligned}
$$

Now, either we have to continue to calculate equations for the triples, which will involve even higher clusters, or we can try to approximate higher moments by lower ones. The simplest scheme is the mean field approximation, leading to a closed system of ODEs for the total number of infecteds, recovered and susceptibles only. For the present system this mean field approximation was analysed previously [5]. Here we go one step beyond by approximating the triples into pairs. There is a vast literature on pair approximation, with one of the first applications to dynamics by Dickman in 1986 [11] and one of the first to population biology by Matsuda et al. in 1992 [12]. For a good recent summary see, e.g., [13]. 


\section{Pair approximation}

To obtain approximate expressions for the triples appearing in the equation system (8), we consider only the true triples $\widetilde{\langle I S I\rangle}$, where the last site of, e.g., infected is not identical with the first, hence with the definition

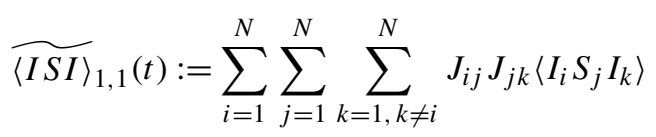

we have as relation with the definition in Eq. (7)

$$
\langle I S I\rangle=\widetilde{\langle I S I\rangle}+\langle S I\rangle
$$

when the local variable at site $k$, here $I_{k}$, is of the same type as the one in $i$, here $I_{i}$ and simply

$$
\langle S I R\rangle=\widetilde{\langle S I R\rangle}
$$

when the local variable at site $k$, now $R_{k}$, is different from the one in $i$, now $S_{i}$. For triples, which are by nature just pairs, i.e., with $i=k$, we have locally $\left\langle I_{i} S_{j} I_{k}\right\rangle=\left\langle I_{i}^{2} S_{j}\right\rangle=\left\langle I_{i} S_{j}\right\rangle$, since $I_{i} \in\{0,1\}$, so they should be counted as pairs, i.e., given by Eq. (10), whereas in Eq. (11) for $i=k$ we have $\left\langle S_{i} I_{j} R_{k}\right\rangle=\left\langle S_{i} I_{j} R_{i}\right\rangle=0$, since $S_{i}, R_{i} \in\{0,1\}$ and $S_{i}+I_{i}+R_{i}=1$. The difference between $\widetilde{\langle I S I\rangle}$ and $\langle I S I\rangle$ does first appear in the triples, since in the pairs the diagonal of the adjacency matrix is zero, avoiding the eventual double counting of singlets.

Then we consider all the possible combinations, where sums over the adjacency matrix only come to play $\sum_{j=1}^{N} J_{i j}=: Q_{i}$. These indicate the number of neighbours to a lattice site $i$, and from now on we will only consider regular lattices (later the square lattice with periodic boundary conditions). Hence we can assume that all $Q_{i}$ are equal, i.e., $Q=Q_{i}$ for all $i$.

The pair approximation yields

$$
\widetilde{\langle S I R\rangle} \approx \frac{Q-1}{Q} \cdot \frac{\langle S I\rangle \cdot\langle I R\rangle}{\langle I\rangle}
$$

obtained from an analog for the Bayesian formula for conditional probabilities applied to the local expectation values

$$
\left\langle S_{i} I_{j} R_{k}\right\rangle \approx \frac{\left\langle S_{i} I_{j}\right\rangle \cdot\left\langle I_{j} R_{k}\right\rangle}{\left\langle I_{j}\right\rangle}
$$

and a spatial homogeneity argument, namely

$$
\left\langle I_{j} R_{k}\right\rangle \approx\left\langle I_{i} R_{j}\right\rangle \approx \frac{\langle I R\rangle}{N Q}
$$

and

$$
\left\langle I_{i}\right\rangle \approx \frac{\langle I\rangle}{N} .
$$

With expressions like the one in Eq. (12) we obtain a closed equation system for the dynamics (8). To simplify this equation system further we now consider additional balance equations.

\subsection{Balance equations for means and pairs}

From $S_{i}+I_{i}+R_{i}=1$ it follows immediately that for the means

$$
\langle S\rangle+\langle I\rangle+\langle R\rangle=N
$$

holds, and from this that $\frac{d}{d t} N=0=\frac{d}{d t}\langle S\rangle+\frac{d}{d t}\langle I\rangle+\frac{d}{d t}\langle R\rangle$ also holds. A check of the results of the dynamics (4) is to insert the three equations and verify the sum to be equal to zero. In this case it can be confirmed by eye immediately.

For the pair dynamics in all variables $S, I$ and $R$, however, the check of the balance is not so obvious. The balance equation is now, again for regular lattices,

$$
\langle S S\rangle+\langle I I\rangle+\langle R R\rangle+2\langle S I\rangle+2\langle S R\rangle+2\langle I R\rangle=N \cdot Q
$$

which can be obtained by explicitly expressing all terms including variable $S$ in terms of the independent variables $I$ and $R$, hence

$$
\langle S R\rangle+\langle I R\rangle+\langle R R\rangle=Q\langle R\rangle,
$$

etc. The pair balance dynamics now follows from considering $\frac{d}{d t}(N \cdot Q)$ from Eq. (18) which is exactly fulfilled by the ODE system for the pair dynamics as given above. From these balance equations we can reduce the ODE system for total expectation values and for pair expectation values to five independent variables $\langle I\rangle,\langle R\rangle,\langle S I\rangle,\langle R I\rangle$ and $\langle S R\rangle$. 


\subsection{Pair approximation equations in reduced variables}

In independent variables we obtain the following closed ODE system using pair approximations

$$
\begin{aligned}
& \frac{d}{d t}\langle I\rangle=\beta\langle S I\rangle-\gamma\langle I\rangle+\tilde{\beta}\langle R I\rangle, \\
& \frac{d}{d t}\langle R\rangle=\gamma\langle I\rangle-\alpha\langle R\rangle-\tilde{\beta}\langle R I\rangle, \\
& \frac{d}{d t}\langle S I\rangle=\alpha\langle R I\rangle-(\gamma+\beta)\langle S I\rangle+\beta(Q-1)\langle S I\rangle-\beta \frac{Q-1}{Q} \frac{(2\langle S I\rangle+\langle S R\rangle) \cdot\langle S I\rangle}{N-\langle I\rangle-\langle R\rangle}+\tilde{\beta} \frac{Q-1}{Q} \frac{\langle S R\rangle\langle R I\rangle}{\langle R\rangle}, \\
& \frac{d}{d t}\langle R I\rangle=\gamma(Q\langle I\rangle-\langle S I\rangle)-(\alpha+2 \gamma+\tilde{\beta})\langle R I\rangle+\beta \frac{Q-1}{Q} \frac{\langle S R\rangle\langle S I\rangle}{N-\langle I\rangle-\langle R\rangle}+\tilde{\beta} \frac{Q-1}{Q} \frac{(Q\langle R\rangle-\langle S R\rangle-2\langle R I\rangle) \cdot\langle R I\rangle}{\langle R\rangle}, \\
& \frac{d}{d t}\langle S R\rangle=\gamma\langle S I\rangle+\alpha(Q\langle R\rangle-2\langle S R\rangle-\langle R I\rangle)-\beta \frac{Q-1}{Q} \frac{\langle S R\rangle\langle S I\rangle}{N-\langle I\rangle-\langle R\rangle}-\tilde{\beta} \frac{Q-1}{Q} \frac{\langle R I\rangle\langle S R\rangle}{\langle R\rangle},
\end{aligned}
$$

which is the basis of the analysis following now.

\section{The critical points and phase transition lines of the SIRI model}

We investigate the stationary states of the closed equations system (19) in order to obtain the phase transition lines which have been described in stochastic simulations of simpler time discrete models [3] and also considered for the present basic reinfection model in mean field approximation [5].

\subsection{Stationary states of the SIRI model}

The full SIRI system cannot be solved analytically at stationarity. After some simplifications, expressing $\langle R I\rangle^{*},\langle S I\rangle^{*}$ and $\langle S R\rangle^{*}$ as functions of the variables $\langle I\rangle^{*}$ and $\langle R\rangle^{*}$ only, we are left with two implicit equations for the remaining variables $\langle I\rangle^{*}$ and $\langle R\rangle^{*}$. Explicitly, we obtain the following equations:

From the ODE system (19), second equation, at stationarity, giving $0=\frac{d}{d t}\langle R\rangle^{*}=\gamma\langle I\rangle^{*}-\alpha\langle R\rangle^{*}-\tilde{\beta}\langle R I\rangle^{*}$ we obtain directly $\langle R I\rangle^{*}$ as function of $\langle I\rangle^{*}$ and $\langle R\rangle^{*}$, hence

$$
\langle R I\rangle^{*}=\frac{\gamma}{\tilde{\beta}}\langle I\rangle^{*}-\frac{\alpha}{\tilde{\beta}}\langle R\rangle^{*} .
$$

From $0=\frac{d}{d t}\langle I\rangle^{*}=\beta\langle S I\rangle^{*}-\gamma\langle I\rangle^{*}+\tilde{\beta}\langle R I\rangle^{*}$ we obtain using Eq. (20)

$$
\langle S I\rangle^{*}=\frac{\alpha}{\beta}\langle R\rangle^{*} .
$$

Further from $0=\frac{d}{d t}\langle S R\rangle^{*}$ we obtain

$$
\langle S R\rangle^{*}=\frac{\alpha Q\langle R\rangle^{*}-\alpha\langle R I\rangle^{*}+\gamma\langle S I\rangle^{*}}{2 \alpha+\frac{Q-1}{Q}\left(\beta \frac{\langle S I\rangle^{*}}{N-\langle I\rangle^{*}-\langle R\rangle^{*}}+\tilde{\beta} \frac{\langle R I\rangle^{*}}{\langle R\rangle^{*}}\right)},
$$

which is explicitly also expressible as function of $\langle I\rangle^{*}$ and $\langle R\rangle^{*}$ only.

But from

$$
0=\frac{d}{d t}\langle R I\rangle^{*}=: f\left(\langle I\rangle^{*},\langle R\rangle^{*}\right)
$$

and

$$
0=\frac{d}{d t}\langle S I\rangle^{*}=: g\left(\langle I\rangle^{*},\langle R\rangle^{*}\right)
$$

we only get implicit equations for the variables $\langle I\rangle^{*}$ and $\langle R\rangle^{*}$. We will first consider some special cases where the above system can be solved analytically very easily, namely the limiting case for reinfection rate equal to first infection rate (the SIS limit of the SIRI model), then vanishing reinfection rate (the SIR limit of the SIRI model), and finally the limit of vanishing transition from recovered to susceptible $\alpha$. In these cases we can give the stationary values $\langle I\rangle^{*}$, etc., as well as the critical parameters respectively critical line. For the general case, Eqs. (20)-(24), we finally can calculate in the limit near criticality via a scaling argument the critical line. No general solution for the total number of infected, etc., at stationarity can be given. 


\subsection{The $\tilde{\beta}=\beta$ limit or SIS limit}

The ODE system Eq. (19) can be treated analytically to obtain the stationary solution $\langle I\rangle^{*}$ as a function of the parameters in various situations, e.g., for $\tilde{\beta}=\beta$. This is the limit in which the SIRI system (19) behaves at stationarity like an SIS system.

When $\tilde{\beta}=\beta$, there is no difference any more between recovered and the susceptibles, hence we can add the recovered individuals to the susceptibles and treat the SIRI model as an SIS model with infection rate $\beta$ and recovery rate $\gamma$. Now, we only need to consider the dynamics of $\langle I\rangle$ and $\langle S I\rangle$ which is given, under pair approximation, by the following equations

$$
\begin{aligned}
& \frac{d}{d t}\langle I\rangle=\beta\langle S I\rangle-\gamma\langle I\rangle, \\
& \frac{d}{d t}\langle S I\rangle=\gamma Q\langle I\rangle+(\beta(Q-2)-2 \gamma)\langle S I\rangle-2 \beta \frac{Q-1}{Q} \frac{\langle S I\rangle^{2}}{N-\langle I\rangle} .
\end{aligned}
$$

From this we can calculate the stationary value of infected, giving either the trivial disease free state $\langle I\rangle_{1}^{*}=0$ or the endemic state

$$
\langle I\rangle_{2}^{*}=N \frac{Q(Q-1) \beta-Q \gamma}{Q(Q-1) \beta-\gamma}
$$

and then for the SIS limit the critical value for $\beta$ is given by

$$
\beta_{c}=\frac{\gamma}{Q-1}
$$

as was calculated previously in Ref. [6] for the SIS epidemics. The resulting critical point is shown in Fig. 1 and lies along the line where $\tilde{\beta}=\beta$ in Fig. 2, the SIS limiting case.

\subsection{The $\alpha=0$ limit}

Considering the stationary state equations (20) to (24) in Section 4.1 in the special case of $\alpha=0$ we obtain

$$
\langle R I\rangle^{*}=\frac{\gamma}{\tilde{\beta}}\langle I\rangle^{*}
$$

and

$$
\langle S I\rangle^{*}=0, \quad\langle S R\rangle^{*}=0,
$$

so that also $\langle S\rangle^{*}=0$. Hence $\langle R\rangle^{*}=N-\langle I\rangle^{*}$.

Eq. (24) in the limit $\alpha=0$ becomes identical $0=0$, and the remaining equation (23) becomes

$$
\gamma Q\langle I\rangle^{*}-(2 \gamma+\tilde{\beta})\langle R I\rangle^{*}+\tilde{\beta} \frac{Q-1}{Q} \frac{\left(Q\langle R\rangle^{*}-2\langle R I\rangle^{*}\right) \cdot\langle R I\rangle^{*}}{\langle R\rangle^{*}}=0
$$

and inserting the above equations, Eqs. (27) and (28), gives

$$
\gamma Q\langle I\rangle^{*}-(2 \gamma+\tilde{\beta}) \frac{\gamma}{\tilde{\beta}}\langle I\rangle^{*}+\gamma \frac{Q-1}{Q} \frac{\left(Q N-Q\langle I\rangle^{*}-2 \frac{\gamma}{\tilde{\beta}}\langle I\rangle^{*}\right) \cdot\langle I\rangle^{*}}{N-\langle I\rangle^{*}}=0
$$

an equation which is independent of $\beta$. It has as one stationary state $\langle I\rangle_{1}^{*}=0$. The remaining equation

$$
\gamma Q-(2 \gamma+\tilde{\beta}) \frac{\gamma}{\tilde{\beta}}+\gamma \frac{Q-1}{Q} \frac{\left(Q N-Q\langle I\rangle^{*}-2 \frac{\gamma}{\tilde{\beta}}\langle I\rangle^{*}\right)}{N-\langle I\rangle^{*}}=0
$$

gives the solution for $\langle I\rangle_{2}^{*}$. It is explicitly after some calculation

$$
\langle I\rangle_{2}^{*}=N \frac{Q(Q-1) \tilde{\beta}-Q \gamma}{Q(Q-1) \tilde{\beta}-\gamma}
$$

which is the solution of the SIS pair approximation dynamics at stationarity equation (25).

From Eq. (31) directly (or from Eq. (32)) the critical value $\tilde{\beta}_{c}$ for the parameter $\tilde{\beta}$ can be calculated considering the condition that $\langle I\rangle_{2}^{*} \rightarrow\langle I\rangle_{1}^{*}=0$, hence setting $\langle I\rangle_{2}^{*}=0$. The result is

$$
\tilde{\beta}_{c}=\frac{\gamma}{Q-1}
$$

independently of the parameter $\beta$. This solution (33) gives a straight horizontal line in the parameter phase diagram for $\beta$ and $\tilde{\beta}$, as shown in Fig. 2. 


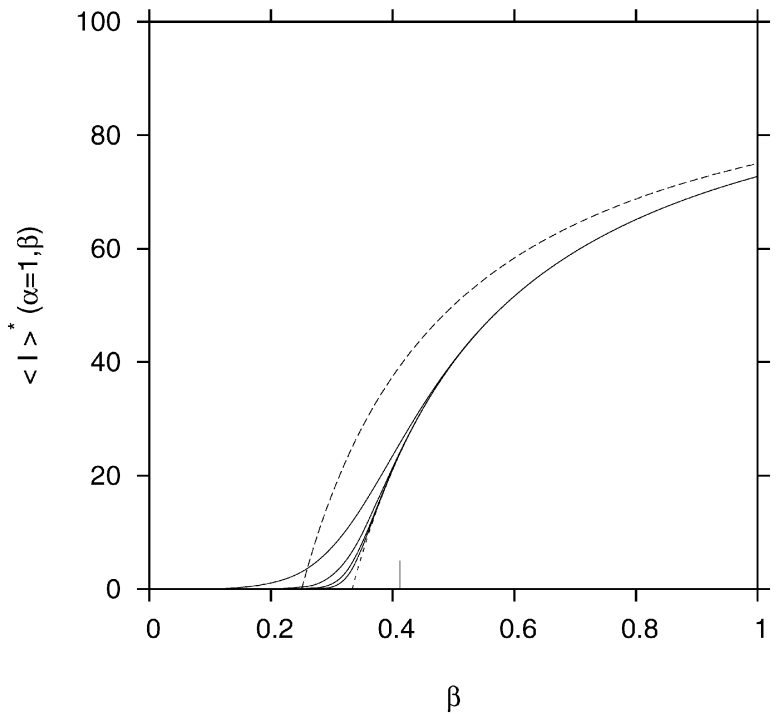

Fig. 1. For $\tilde{\beta}=\beta$ (the SIS limit) we compare the mean field solution (isolated dashed line) with the pair approximation (dotted line) for $\langle I\rangle^{*}(\beta)$ and the convergence of the time dependent solution of the SIRI pair dynamics (straight lines for various stopping times $t_{\max }$ approximating the dotted line). Further the critical value for the contact process is given as well, as obtained from extended spatial stochastic simulation reported in the literature [14] as $\beta_{c}=0.4122\left(=\lambda_{c} / 4\right.$ in [14]). The pair approximation solution approaches the simulation value better than the mean field solution. (Parameters $Q=4$ appropriate for spatial two dimensional systems, $\gamma=1$ throughout the figures, here also $N=100$.)

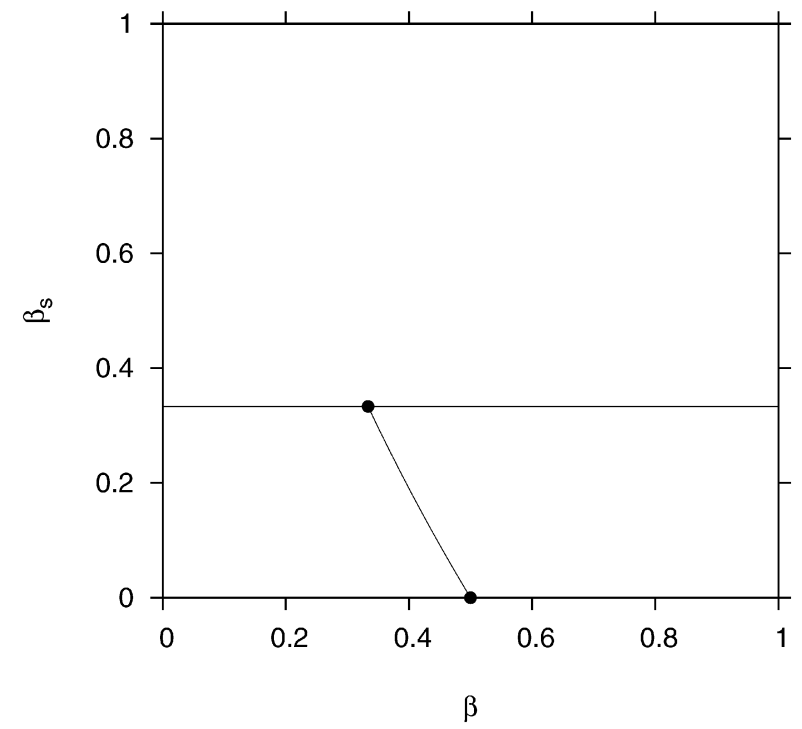

Fig. 2. The phase transition point for $\tilde{\beta}=\beta$ as given in pair approximation in Eq. (26) in the SIS limiting case is the dot on the diagonal $\tilde{\beta}=\beta$. The phase transition line for $\alpha=0$ obtained in pair approximation, according to Eq. (33) is the horizontal line through that point. The critical point for $\tilde{\beta}=0$ (the SIR-limit) obtained in pair approximation Eq. (36) and the phase transition line between no-growth and ring-growth determined from the analytic solution for the $\alpha=0$ case which is explicitly given in Eq. (44) connecting the two points are shown.

\subsection{The $\tilde{\beta}=0$ limit or SIR limit}

In analogy to Section 4.3 we can calculate from the equation system (19) the stationary state solution for the limit $\tilde{\beta}=0$. The stationary state $\langle I\rangle_{1}^{*}=0$ can be found and the other stationary state follows from an equation of the form

$$
a_{2}\left(\langle I\rangle_{2}^{*}\right)^{2}+a_{1}\langle I\rangle_{2}^{*}+a_{0}=0
$$

with the coefficients

$$
\begin{aligned}
a_{2}= & \beta Q^{2}(Q-1)\left(\alpha^{3}+2 \alpha^{2} \gamma+2 \alpha \gamma^{2}+\gamma^{3}\right)-Q \gamma\left(\alpha^{3}+\gamma^{3}\right)-(\alpha+\gamma)(2 \alpha \gamma Q+\beta Q(\alpha+\gamma)+\alpha \gamma) \gamma, \\
a_{1}= & -\alpha \beta Q^{2} N\left[2 \alpha^{2}(Q-1)+3 \gamma(Q \alpha-\gamma)+2 \gamma(Q \gamma-2 \alpha)\right] \\
& +\alpha \gamma Q N\left[Q\left(\alpha^{2}+\alpha \gamma+\gamma^{2}\right)+\beta(\alpha+\gamma)+\left(\alpha^{2}+3 \alpha \gamma+\gamma^{2}\right)\right], \\
a_{0}= & \alpha^{2} Q^{2} N^{2}(\alpha \beta(Q-1)+\beta \gamma(Q-2)-\gamma(\alpha+\gamma)) .
\end{aligned}
$$

Setting $\langle I\rangle_{2}^{*}=0$ (which is the same as $a_{0}=0$ ), like shown in Section 4.3, gives the explicit solution for the critical value $\beta_{c}$ as

$$
\beta_{c}=\frac{\gamma+\alpha}{Q-2+(Q-1) \frac{\alpha}{\gamma}}
$$

and in the limit of $\alpha=0$,

$$
\beta_{c}=\frac{\gamma}{Q-2}
$$

in agreement with previously reported results [7]. The solution of the critical value $\beta_{c}=\frac{\gamma}{Q-2}$ is shown in the phase diagram for $\beta$ and $\tilde{\beta}$ in Fig. 2.

\subsection{Numerical integration of the pair approximation dynamic system}

We numerically integrate the ODE system (19) for fixed $\gamma:=1$ and small $\alpha:=0.05$, varying the infection rates $\beta$ and $\tilde{\beta}$. From the simulations analogously to Figs. 3 to 5 we can determine the critical line for a small but finite $\alpha$ value between the no-growth and the annular growth region in pair approximation. 


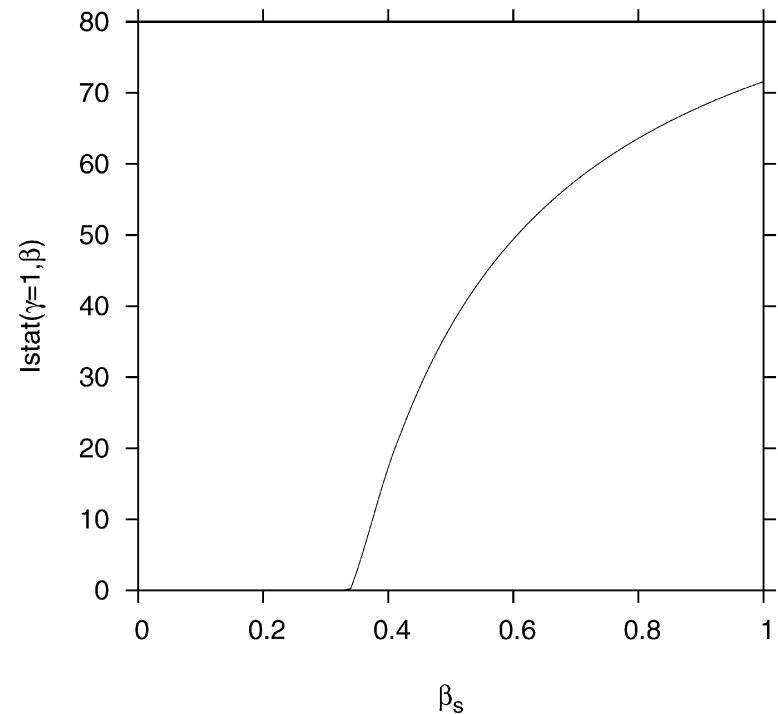

(a)

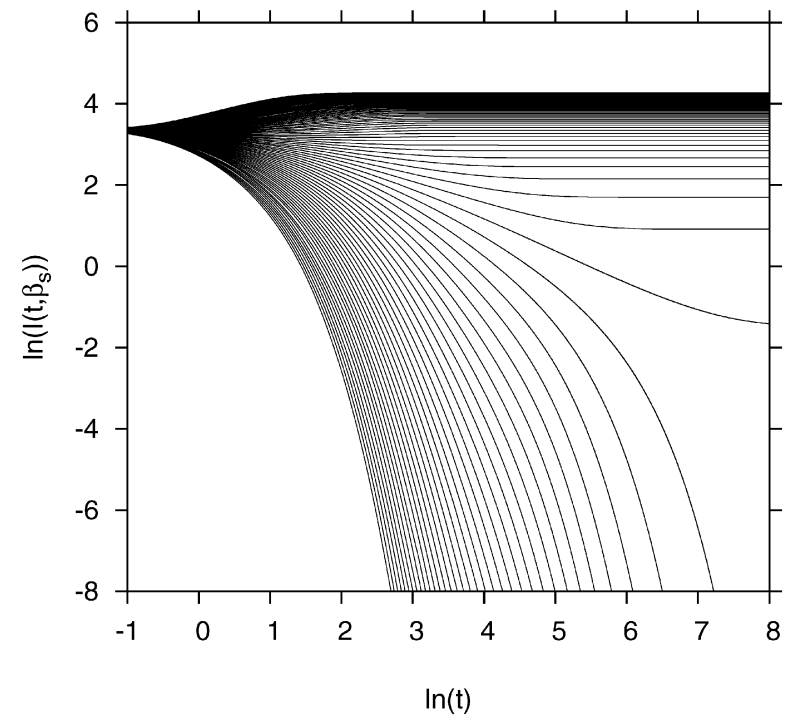

(b)

Fig. 3. (a) Integrating the system (19) numerically up to time $t_{\max }$ gives $I\left(t_{\max }\right)$ with changing $\tilde{\beta}$ for $\beta=\gamma /(Q-1)$ the SIS critical point value. (b) The logarithm of $I(t), \ln (I(t))$ versus $\ln (t)$ for various $\tilde{\beta}$ values. A clear distinction is visible for the sub-critical versus (going towards minus infinity) the supercritical values (going to finite values at $t_{\max }$ ).

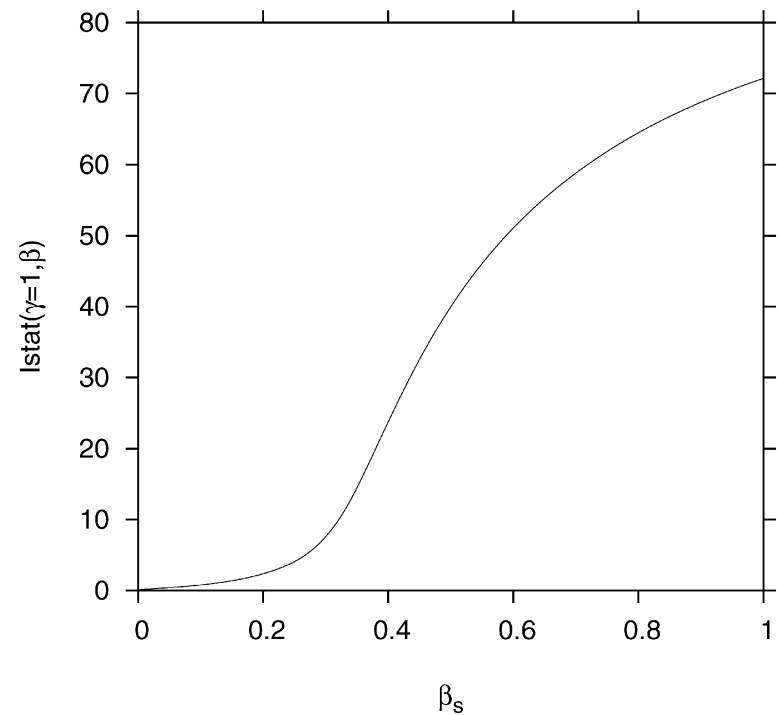

(a)

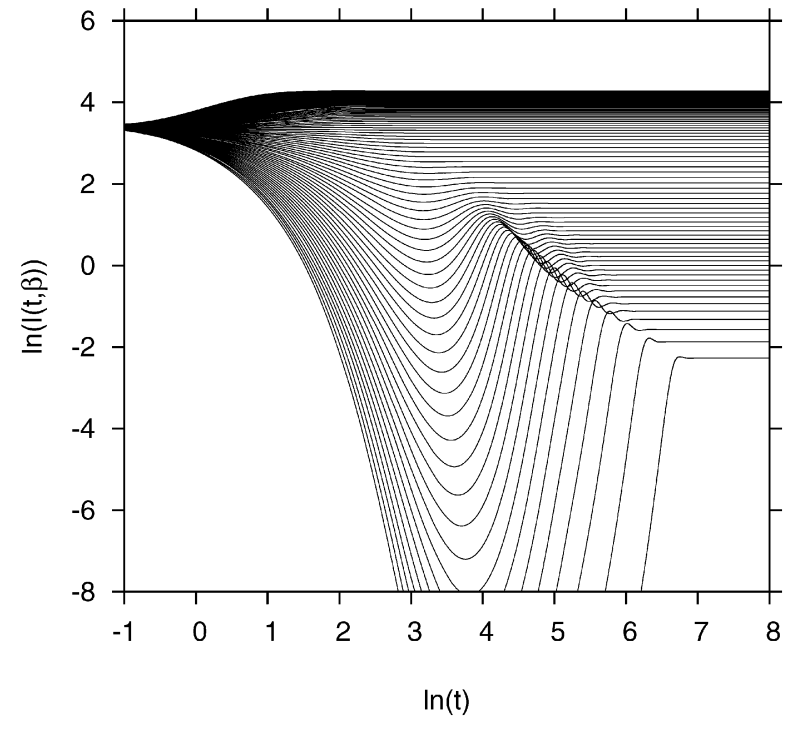

(b)

Fig. 4. (a) $I\left(t_{\max }\right)$ for $\beta=\gamma /(Q-2)$ the SIR critical point value in the limit $\alpha=0$. However the numerics are done for small but finite $\alpha=0.05$. (b) $\ln (I(t))$ versus $\ln (t)$ for various $\tilde{\beta}$ values. For all $\tilde{\beta}$ values the curves finally go to finite values, none towards minus infinity, hence all $\tilde{\beta}$ values are supercritical.

We can determine for small values of $\alpha$ from the numeric solutions of the SIRI pair dynamics (19) directly the critical values. The result is shown in Fig. 6 as a line between the SIS limiting critical point and the SIR limiting critical point. The numerical integration for Fig. 6 have $\alpha=0.05$ as small $\alpha$ value. The SIRI pair dynamics, Eq. (19), is varied between $\beta=\gamma /(Q-1)$ and $\beta=\gamma /(Q-2)$, then the critical value for $\tilde{\beta}$ is determined for each value of $\beta$.

\subsection{Analytic expression}

We investigate Eqs. (20)-(24) further, using the information that when $\langle I\rangle^{*}$ goes to zero, so does $\langle R\rangle^{*}$, but the quotient stays finite

$$
\lim _{\langle I\rangle^{*} \rightarrow 0} \frac{\langle R\rangle^{*}}{\langle I\rangle^{*}}=\frac{\gamma \cdot B}{2 \alpha \cdot E}
$$




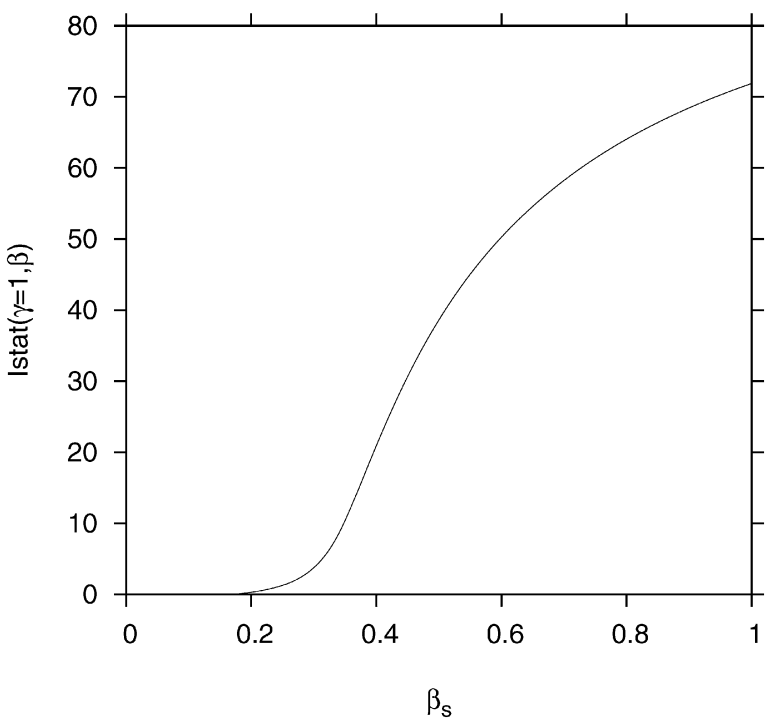

(a)

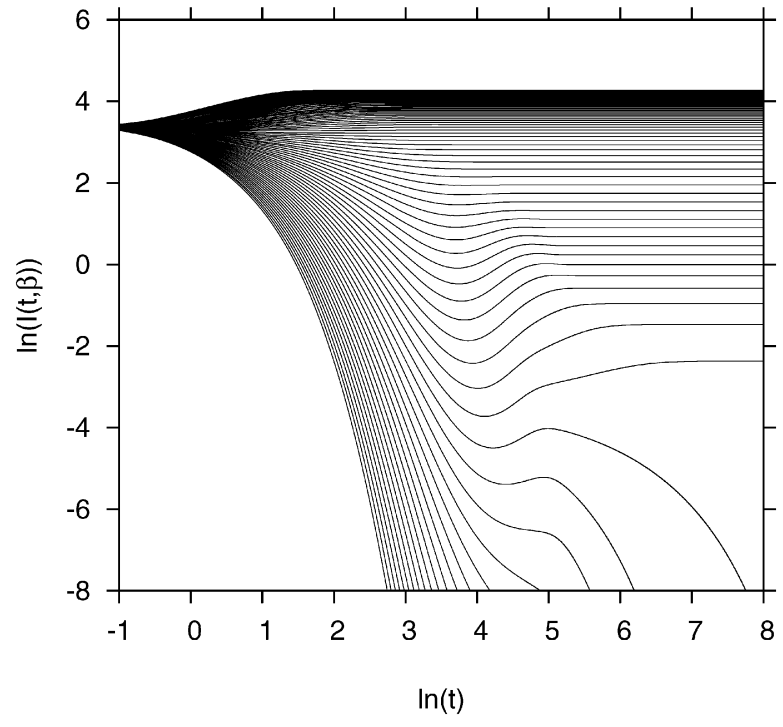

(b)

Fig. 5. (a) $I\left(t_{\max }\right)$ for $\beta=\gamma /(Q-1.5)$, i.e., between the values for the SIS critical point value $\beta=\gamma /(Q-1)$ and the SIR critical point value $\beta=\gamma /(Q-2)$. (b) $\ln (I(t))$ versus $\ln (t)$ for various $\tilde{\beta}$ values. Sub-critical and super-critical values for $\tilde{\beta}$ can be distinguished finally, but initially some of the supercritical curves go to very low numbers, which for smaller $t_{\max }$ could be mistaken as sub-critical.

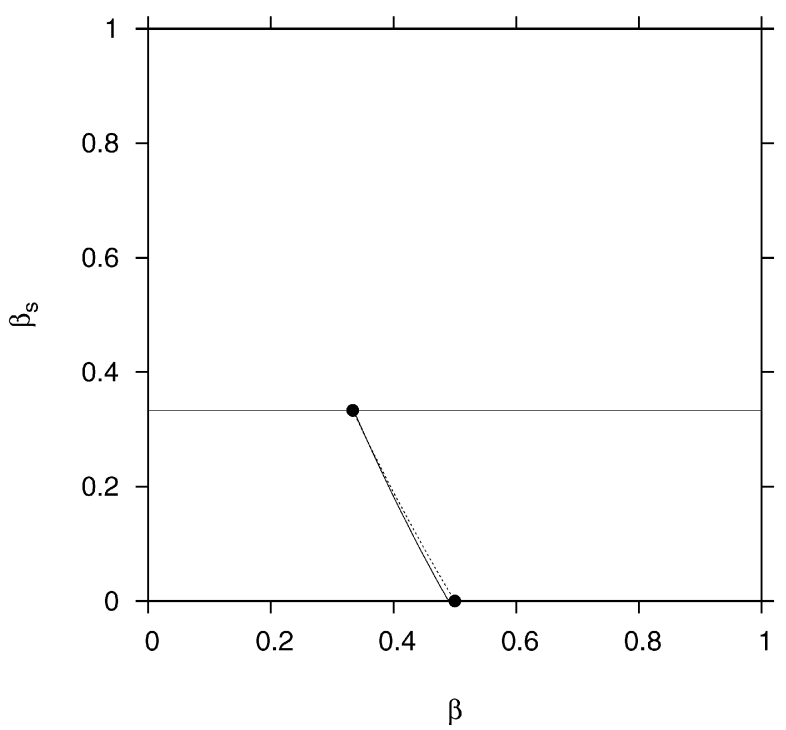

Fig. 6. Comparison for the phase transition line between no-growth and ring-growth determined numerically for small but finite $\alpha=0.05$, straight line, and the analytic solution for the $\alpha=0$ case, dotted line. The analytic curve for finite $\alpha=0.05$ agrees completely with the simulations.

(with $B$ and $E$ given below). Only later performing the limit of $\alpha \rightarrow 0$, we obtain the following solution for the curve $\beta(\tilde{\beta})$ for general $\gamma$ and also non-vanishing $\alpha$ as

$$
\beta(\tilde{\beta})=\frac{C(\tilde{\beta})}{D(\tilde{\beta})}
$$

with the expressions

$$
A:=\sqrt{(Q-1)^{2} \tilde{\beta}^{2}+2 Q(Q-1)(\alpha-\gamma) \tilde{\beta}+Q^{2}(\alpha+\gamma)^{2}}
$$

and

$$
B:=\alpha+Q \gamma-(Q-1)(\alpha+\tilde{\beta})+A
$$

and numerator and denominator of Eq. (38), first the numerator

$$
C=Q \gamma \tilde{\beta} \cdot B^{2}
$$


respectively with

$$
E:=\alpha+\tilde{\beta}+Q \gamma
$$

the denominator

$$
D=(Q \gamma-(Q-1) \alpha) \cdot B^{2}+2(Q(Q-1) \tilde{\beta}+2(Q-1) \alpha-Q \gamma) \cdot E \cdot B-4(Q-1) \alpha \cdot E^{2} .
$$

This complets the expression for the critical curve $\beta(\tilde{\beta})$ for the general $\alpha$ and $\gamma$ case. For the limit of $\alpha \rightarrow 0$ we obtain again a rather simple expression

$$
\beta(\tilde{\beta})=\frac{\gamma^{2} Q-\gamma \tilde{\beta}(Q-1)}{\gamma Q(Q-2)+\tilde{\beta}(Q-1)}
$$

shown graphically in Fig. 2. We also tested the general $\alpha$ case and the general $\gamma$ case against the numerical solutions given above with success (not shown here, since the curves lie exactly on top of each other inside the graphical resolution).

We finally show the finite $\alpha=0.05$ case in comparison with the $\alpha=0$ curve, finding only small differences (Fig. 6). So the numerical procedure shown above gives a rather good impression of the phase diagram in the limiting but numerically difficult to access case of vanishing $\alpha$ as expected. This is a good sign for future studies of purely stochastic simulations, which close to criticality are expected to be rather time consuming.

\section{Summary}

We have calculated the phase diagram for the basic reinfection model SIRI analytically in the pair approximation frame work and confirmed the results with simulations. The limiting cases of the SIS and SIR epidemics agree with previously reported results in the literature.

\section{Acknowledgements}

We would like to thank Peter Grassberger, Jülich and Calgary, for pointing our interest to the physics literature on partial immunization models, and Gabriela Gomes, Lisbon, for discussions and hints on the reinfection literature in the epidemiology community. Further thanks to Minus van Baalen, Paris, and Vincent Jansen, London, for discussions on various aspects of the present manuscript and Ronald Dickman, Belo Horizonte, for additional literature references.

\section{References}

[1] M.G.M. Gomes, L.J. White, G.F. Medley, J. Theor. Biol. 228 (2004) 539.

[2] M.G.M. Gomes, L.J. White, G.F. Medley, J. Theor. Biol. 236 (2005) 111.

[3] P. Grassberger, H. Chaté, G. Rousseau, Phys. Rev. E 55 (1997) 2488.

[4] S.M. Dammer, H. Hinrichsen, J. Stat. Mech.: Theor. Exp. (2004) P07011, 17.

[5] N. Stollenwerk, D. Gökaydin, F. Hilker, S. van Noort, G. Gomes, The reinfection threshold corresponds to the mean field version of the transition between annular and compact growth in spatial epidemic models (2007), submitted for publication.

[6] S.A. Levin, R. Durrett, Philos. Trans. R. Soc. London, Ser. B 351 (1996) 1615.

[7] J. Joo, J.L. Lebowitz, Phys. Rev. E 70 (2004) 036114.

[8] R. Breban, S. Blower, J. Theor. Biol. 235 (2005) 151.

[9] N.G. van Kampen, Stochastic Processes in Physics and Chemistry, North-Holland, Amsterdam, 1992.

[10] R.J. Glauber, J. Math. Phys. 4 (1963) 294.

[11] R. Dickman, Phys. Rev. A 34 (1986) 4246.

[12] H. Matsuda, N. Ogita, A. Sasaki, K. Satō, Prog. Theor. Phys. 88 (1992) 1035.

[13] D.A. Rand, Correlation equations and pair approximations for spatial ecologies, in: J. McGlade (Ed.), Advanced Ecological Theory, Blackwell Science, Oxford, 1999, pp. 100-142.

[14] R. Dickman, J.K. da Silva, Phys. Rev. E 58 (1998) 4266. 